Chapter 9

\title{
System Safety of Gas Turbines: Hierarchical Fuzzy Markov Modelling
}

\author{
G. G. Kulikov, V. Yu. Arkov and A.I. Abdulnagimov \\ Additional information is available at the end of the chapter \\ http://dx.doi.org/10.5772/54443
}

\section{Introduction}

Reliability, safety and durability represent important properties of modern aircraft, which is necessary for its effective in-service use.

The reason of the main hazard for aircraft are both random and determined negative influences rendering the controlled object during its use. Faults, failures, disturbances, noises, influences of environment and control errors represent the objectively existing stream of random negative influences on the object.

Statistically, in the recent years the majority of aircraft incidents are connected with the human factor and late fault detection in plane systems. In this regard, requirements to flight safety which demand development of new methods and algorithms of control-and-condition monitoring/ diagnostic for complex objects raise every year. The analysis of modern gas turbine engines has shown that most faults appears in the engine itself and its FADEC (40-75\% for FADEC, Figure 1).

The percentage of faults for FADEC depends on the achieved values for no-failure operation indicators of the engine and FADEC.

During the development of FADEC, it is necessary to adhere to the principles and methods guaranteeing safety and reliability of aircraft in use to guarantee proper responses in all range of negative influences.

Full information on its work is necessary for complete control of a condition of the engine:

1. Reliable detection of a fault cause providing decision-making on a technical condition of gas turbines; 
2. Reliable diagnosis and localization of faults and negative influences are necessary for definition of technical condition of gas turbines for the purpose of providing a reconfiguration and functioning of its subsystems [1,2].

Russian engines

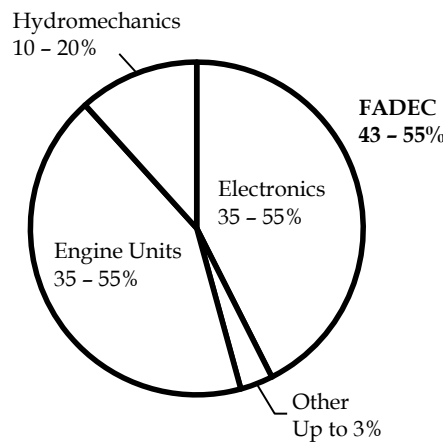

Worldwide statistics

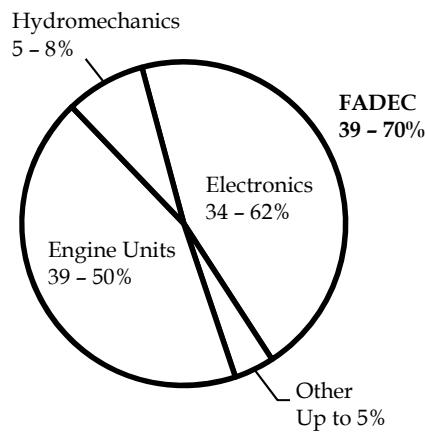

Figure 1. Faults percentage for engines and FADEC

The hardware for condition monitoring of measurement channels in many cases allows to detect only catastrophic (breakage or short circuit) faults, i.e. their stochastic properties on time of the process observed in one object and on a set of objects are not distinguishable [3]. The criteria of warning messages on faults appearance are based mainly on determined logic operations and distinguish between only two conditions: "operational" (fully operational) or "fault". In this chapter, hierarchical fuzzy Markov models for quantitative estimation of system safety of gas turbines taking into account the monitoring of cause-effect relations are considered. Transition from two-valued to fuzzy logic for estimation of degradation indexes and the analysis of fault developments for the gas turbine and its FADEC is considered for this purpose.

\section{Hierarchical model of faults development processes in gas turbines}

Complex diagnostics of the power plant is proposed to be carried out on elements and units, using the hierarchy analysis method [4,5]. First, decomposition into independent subsystems of various hierarchy levels is carried out on structural features. Similarly, the power plant and its systems are represented in the form of hierarchy of elements and blocks.

This approach enables cause-effect relationships to be identified on the hierarchy structure of a system.

In Figure 2, the hierarchical structure of states of the power-plant is shown. The power plant is represented in the form of a hierarchical structure as the complex system consisting of subsystems and elements (units) with built-in test/monitoring functions, according to the distributed architecture. For this purpose, the power plant decomposition might be per- 
formed into independent subsystems with various levels of hierarchy on structural and functional features in the following way:

- Control and monitoring system (FADEC);

- Hydro mechanical system (actuators);

- Fuel system;

- Start-up system;

- Lubricant oil system;

- Drainage system, etc.

The hierarchy analysis allows to utilize the state model on the basis of faults development which enables the system state to be estimated at each level of the hierarchy.

The mathematical model of states is represented as

$$
S=\langle G, F, L, R>\text {, }
$$

where $S$ is state vector,

$G$ is hierarchy of system faults,

$F$ is quantitative estimate of faults,

$L$ is set of fault influence indexes,

$R$ is mutual influence system of faults.

The depth of hierarchy $G$ is referred to as $h$, and $h=0$ for the root element of $G$.

For $G$ the following conditions are satisfied:

1. There is splitting of $G$ into subsets of $h_{k}, k=1 \ldots n$.

2. From $x \in L_{k}$ follows that $x^{-} \subset h_{k+1}, \quad k=1, \ldots, n-1$.

3. From $x \in L_{k}$ follows that $x^{+} \subset h_{k-1}, \quad k=2, \ldots, n$.

For every $x \in G$ there is a weight function such as:

$$
\omega_{x}: x^{-} \rightarrow[0,1] ; \text { where } \sum_{y \in x^{-}} \omega_{x}(y)=1 .
$$

The sets of $h_{i}$ are the hierarchy levels, and function $\omega_{x}$ is a function of fault priority of one level concerning the state of the power-plant $x$. Notice that if $x^{-} \not \subset h_{k+1}$ (for some level of $h_{w}$ ), then $\omega_{x}$ can be defined for all $h_{k}$, if it equals to zero for all faults in $h_{k+1}$ which do not belong to $x^{-}$.

The hierarchical FADEC model integrates:

- functional structure (block diagram);

- physical structure; 


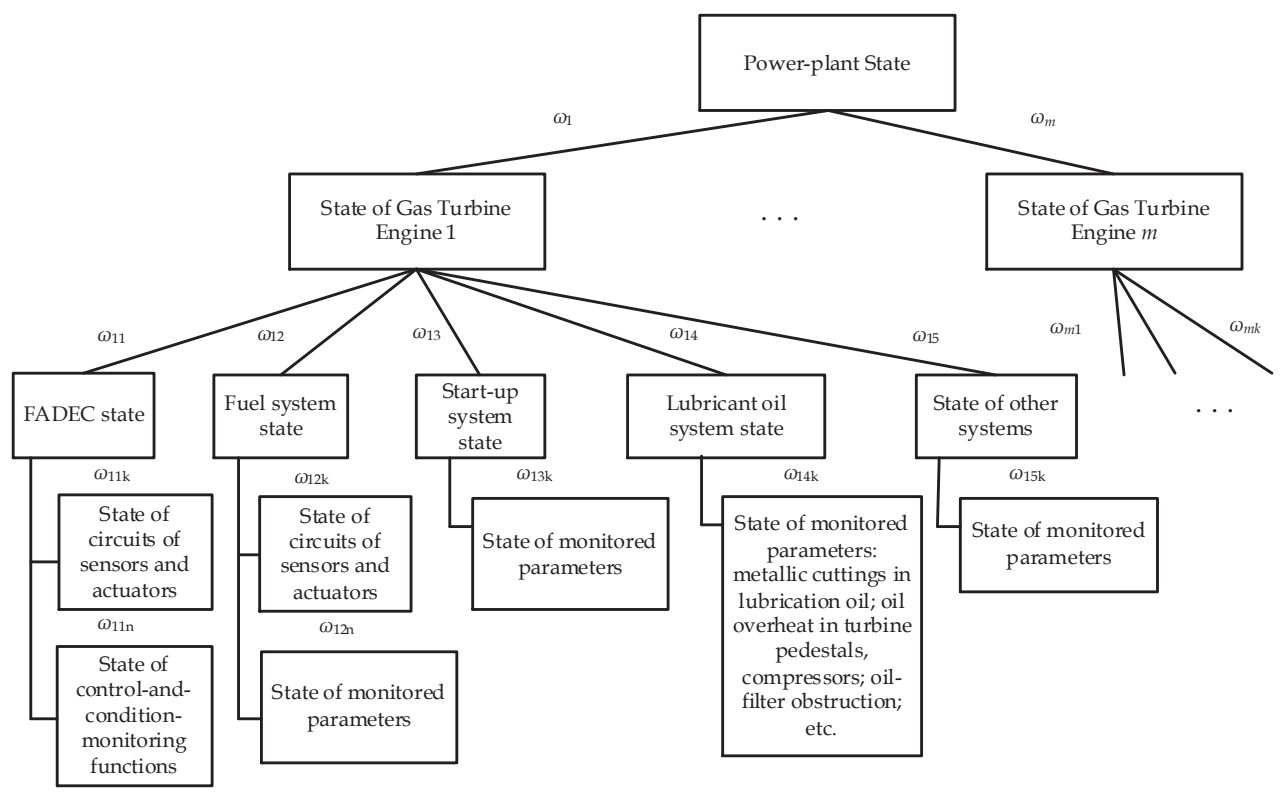

Figure 2. Hierarchical state structure of power plant 


\begin{tabular}{|c|c|c|c|c|}
\hline Fault levels & \multicolumn{2}{|c|}{ Faults } & State & $\begin{array}{c}\text { Fault handling } \\
\text { priority }\end{array}$ \\
\hline \multirow{2}{*}{ Level 1} & \multicolumn{2}{|c|}{ FADEC fault } & \multirow{2}{*}{ Fault } & \multirow{5}{*}{ 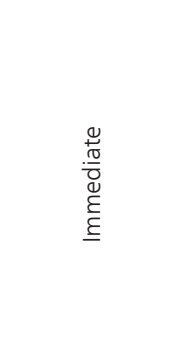 } \\
\hline & Fau & t of lane B & & \\
\hline \multirow{2}{*}{ Levels 2-4 } & & Fault of control function (B) & \multirow{8}{*}{$\begin{array}{l}\frac{1}{0} \\
\stackrel{0}{0} \\
\frac{\pi}{0} \\
\frac{0}{0} \\
0 \\
0 \\
0\end{array}$} & \\
\hline & & Fault of control loop (B) & & \\
\hline Levels 5-6 & $\begin{array}{l}\text { Integrated fault of } \\
\text { measurement for alternative } \\
\text { control law }\end{array}$ & Fault of actuator control circuit (B) & & \\
\hline Level 7 & Fal & t of lane A & & \multirow{4}{*}{$\begin{array}{l}\frac{\varepsilon}{Q} \\
\stackrel{ \pm}{ \pm} \\
\frac{t}{0} \\
\frac{0}{n}\end{array}$} \\
\hline \multirow{2}{*}{ Level 8} & & Fault of control function (A) & & \\
\hline & Fault of control loop & Fault of control loop (A) & & \\
\hline Level 9 & $\begin{array}{l}\text { Integrated fault of } \\
\text { measurement (fault of same } \\
\text { measured parameters in } \\
\text { channels A) }\end{array}$ & $\begin{array}{l}\text { Fault of actuator control circuit (A) } \\
\text { (fault of communication lines of } \\
\text { sensors or fault of FADEC hardware) }\end{array}$ & & \\
\hline \multirow[t]{2}{*}{ Level 10} & $\begin{array}{l}\text { Fault of measurement in } \\
\text { channel (break or short } \\
\text { circuit) }\end{array}$ & & & Long-term \\
\hline & Sensors & Actuators & & \\
\hline
\end{tabular}

Table 1. Fragment of hierarchical classification of faults for FADEC

- tree states (state structure) of elements and units;

- tree of failures influence indexes.

On the hierarchical model, the system of faults interference $R$ with logical operations of a disjunction and a conjunction is applied. Such a system of faults interference allows to analyze the state of all power-plant, both from the bottom up to the top, and from the top down to the bottom and to carry out deeper analysis on various levels of decomposition of the control system using an intermediate state: degradation.

The degradation is understood as "package/complex of degradationary changes of the system" and the degradationary change is "a separately considered irreversible change of a structure of the system, worsening its properties, changing the parameters and characteristics".

Define the main faults of FADEC, the priorities of their elimination and the state they belong to. In Table 1, an exemplary of a fragment of the hierarchical classification of faults for sensors and actuators of FADEC is presented. 
Fault levels 1 through 6 demand immediate handlings and correspond to the "catastrophic" and "critical" states by FAA classification (Federal Aviation Administration, U.S. Department of Transportation), given in [6]. The emerging of such states requires immediate landing of the aircraft. Fault levels 7 through 9 are classified as a "marginal" state and demand operative handling after landing. In this case, it is possible to continue the flight, but postflight repair on the ground is required. Faults at the 10th level demand their handling in long-term prospect.

The degradation process for FADEC starts at the 10th level of hierarchy. From the 4th level of hierarchy, the system starts to approach the system crash that can be regarded as «a critical situation».

Note that development of such faults in certain cases can be detected in advance by estimating the states of elements not only at the level of "0-1" (fully operational, operational/working, fault), but also by considering their gradual degradation.

The state of an element or a system is proposed to be represented in the form of three parameters \{ operational, degradation, fault \}, see Tab. 2.

In the operational state $S=0$, while during fault $S=1$. The degradation degree range from "0" to "1". Thus, the extreme values "0" and "1" are defined according to the determined logic, which is realized in the conventional FADEC (according to the design specifications for the system). The introduction of this intermediate state of "degradation" expands the informativity of the conventional condition monitoring algorithms.

\begin{tabular}{ccc}
\hline Operational & Degradation & Fault \\
\hline$S=0$ & $0<S<1$ & $S=1$ \\
\hline
\end{tabular}

Table 2. Fuzzy representation of state

Based on the faults analysis and the hierarchy of states of the system at each level, the degree of degradation of each item or sub-unit is determined (Figure 3). Fault states are classified via degradation degree as "Negligible", "Marginal", "Critical" and "Catastrophic" [6]. The estimation of the degradation degree is defined on the membership function $S$ which takes values in the range of $S \in[0,1]$.If the degradation degree is closer to " 1 ", the distance to a critical situation will be closer. If the analysis of a system showed that the state vector is $\{0,10,60,3\}$, it is possible to ensure that there is a "distance" before complete fault (a critical situation). As soon as the system state will worsen with the appearance of new faults and will give the following state vector $\{00,30,7\}$, then there will be a distance of 0,3 to a system crash. Thus the most informative indicator will be a tendency of faults appearance (trend), not the existence of degradation itself. Visual trend analysis provides an estimate of time before the critical situations develop and, thus, for early planning of the crew actions $[7,8]$. 


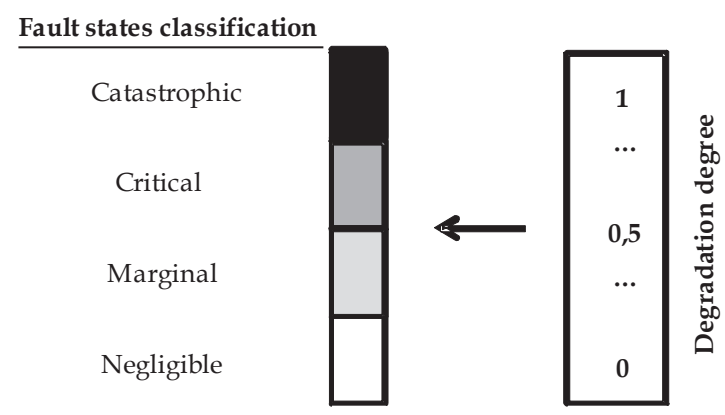

Figure 3. Estimation of "degradation" state

Consider an example of correspondence of degradation degree and the operational state. At the degradation degree of 0,25 , the system is capable to carry out $75 \%$ of demanded functions ( $50 \%$ at 0,5 degradation, $25 \%$ at 0,75 and $0 \%$ at 1 , which is the unavailable state). Such scale allows to define a "threshold" state, below which further operation is not allowed for safety reasons. Using the degradation degree, it is also possible to estimate the distance to a critical situation and the speed of approximation to it (Figure 4).

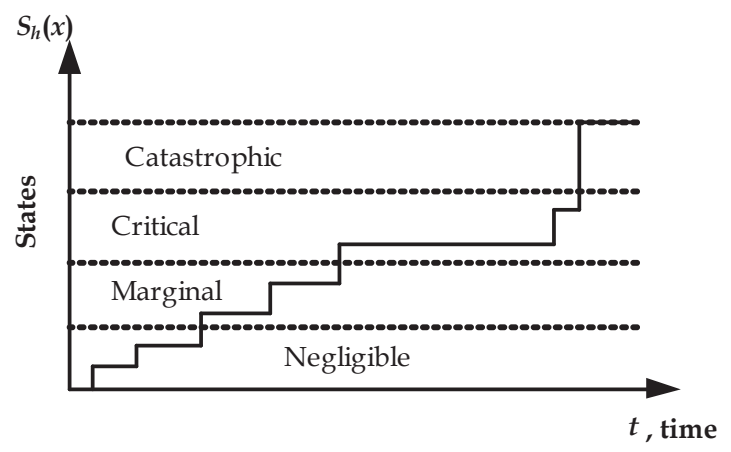

Figure 4. Trend of state dynamics during flight 
Thus, the hierarchical model of fault developments allows to decompose the power-plant on hierarchy levels for obtaining quantitative estimates of the degradation state and gradual faults. The hierarchy analysis allows to utilize the state model and to estimate the system state at each level of hierarchy. The state is represented in the form of a vector with parameters $\{$ operational, degradation, fault $\}$. Depending on the degradation degree it is possible to make an estimation of operability of object and system safety.

\section{Fuzzy technique of determination of state parameters of gas turbine and its systems}

The built-in monitoring system (BMS) is a subsystem of monitoring, diagnosis and classification of faults of the gas turbine and its systems. The fault existence corresponds to a logic state of "1", the absence does to a logic state of "0". Such state classification doesn't allow to establish a "prefault" state, to trace faults' development, and to define degradation of the system and its elements. For more detailed analysis, the estimation of the intermediate state of degradation is proposed. For this purpose, the use of fuzzy logic is considered. Signals from sensors, and also logic state parameters from BMS will transform to linguistic variables during fuzzification to a determined value arrives to the input of the fuzzifier. Let $x$ is the state parameter of an element (for example, the sensor). It is necessary to define fuzzy spaces of input and output variables, and also terms for FADEC sensors. All signals from sensors and actuators will transform into linguistic variables by fuzzification.

Consider an example of fuzzy representation of a state of the two-channel sensor of rotational speed of a high pressure turbocompressor rotor. For monitoring of operational condition of communication lines of the FADEC sensor, a linguistic variable is introduced in the following way:

$$
\Omega=<x_{n}, B=\left(x_{n}\right), U, G, M>\text {, }
$$

where $\Omega_{n}$ is the sensor state,

$x_{n}$ is the number of events when $n$ is beyond the allowed limit band;

$B$ is $\{$ operational, fault $\}$;

$U$ is $[0,4]$

$G$ is the syntactic rule generating terms of set B,

$M$ is the semantic rule, which to each linguistic value $x$ associate with its sense of $M\left(x_{n}\right)$, and $M\left(x_{n}\right)$ designates a fuzzy subset of the carrier $U$.

Say that the sensor is considered failed after the fourth appearance of the shaft speed measurement beyond the allowed boundary, therefore the membership function is formed as shown in Figure 5.

At a single appearance out of limit $\left(x_{n}=1\right)$, membership function $B_{1}$ takes the value 0,7 , and $B_{2}=0,3$. The degradation degree takes the value of membership function $B_{2}$. If the repeated 
breaking the limit doesn't prove to be true during the set period of time, the monitoring algorithm cancels the measurement: $B_{1}=1, B_{2}=0$.

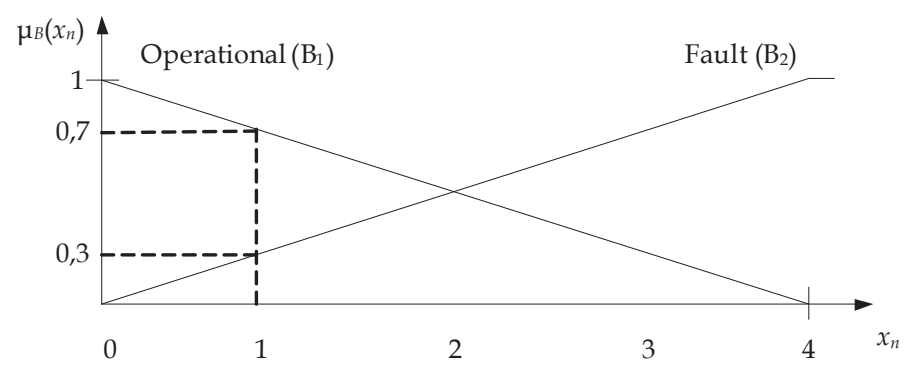

Figure 5. Membership functions of "sensor state"

In Figure 5 two membership functions are shown: state B1 corresponds to the function $\mu_{B 1}$ $\left(x_{n}\right), B_{2}$ is described by the function $\mu_{B 2}\left(x_{n}\right)$.

The way of creating fuzzy rules is presented in Figure 6. This rule base is represented by the table, which is filled in with fuzzy rules as follows [9]:

$$
R^{(1)}: \operatorname{IF}\left(x_{n}=A_{1} \operatorname{AND} x_{n}=B_{1}\right) \text { THEN } y=T_{1}
$$

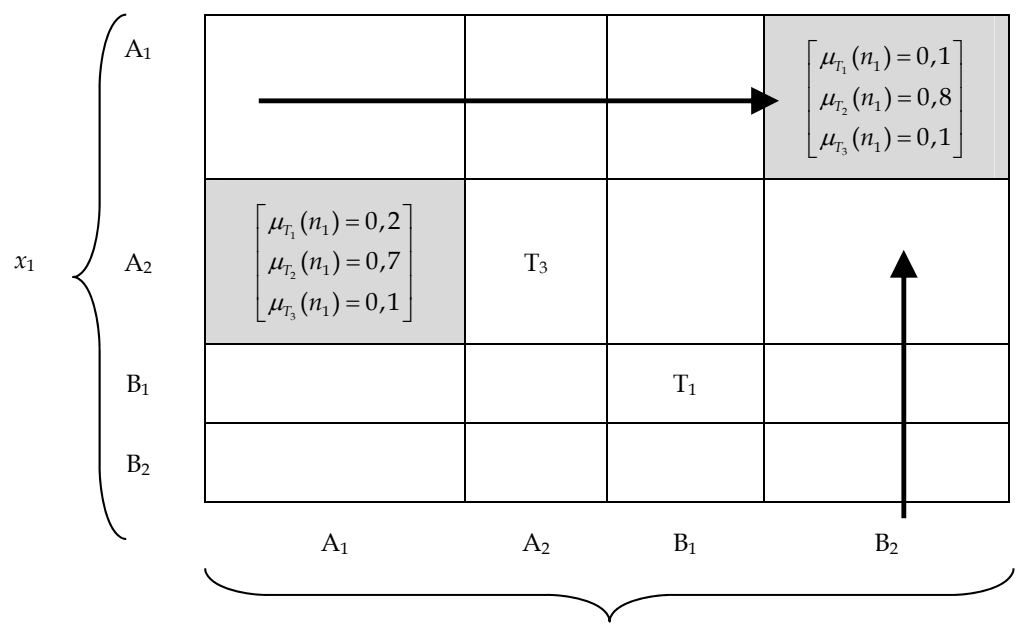

Figure 6. Example of fuzzy rule base 
The values $\mu_{T 1}, \mu_{T 2}, \mu_{T 3}$ are set in the cell at the row "Operational" $\left(A_{1}\right)$ and the column "Fault" $\left(B_{2}\right)$.

Consider a fragment of the rule base for estimates of the sensor state of the low pressure shaft speed. Formulate the first rule: if the 1st coil $\left(n_{11}\right)$ of the sensor is operational $\left(A_{1}\right)$ and the 2 nd coil $\left(n_{12}\right)$ of the sensor is operational $\left(A_{1}\right)$, then the sensor is in the operational condition with the following membership functions:

$$
\left[\mu_{\text {operational }}\left(n_{1}\right)=1 ; \quad \mu_{\text {degradation }}\left(n_{1}\right)=0 ; \quad \mu_{\text {fault }}\left(n_{1}\right)=0\right] .
$$

Write down this rule as follows:

$$
\begin{aligned}
& R^{(1)}: \operatorname{IF}\left(n_{11}=A_{1} \text { AND } n_{12}=A_{1}\right) \text { THEN } y=T_{1} \Rightarrow \\
& {\left[\mu_{T_{1}}\left(n_{1}\right)=1 ; \quad \mu_{T_{2}}\left(n_{1}\right)=0 ; \quad \mu_{T_{3}}\left(n_{1}\right)=0\right] .}
\end{aligned}
$$

Other rules are created in the similar way.

Given a greater number of possible conditions (for example, greater number of the duplicated coils of the sensor), one can develop a discrete-ordered scale of state parameters (Figure 7).

For further analysis of the system, enter the faults influence indexes at each level of hierarchy, using a method of pairwise comparison as it is carried out in the hierarchy analysis method.

Quantitative judgements on the importance of faults are performed for each pair of faults $\left(F_{\mathrm{i}}, F_{j}\right)$ and these are represented by matrix $A$ of the $n \times n$ size.

$$
A=\left(a_{i j}\right), \quad(i, j=1,2,3) .
$$

where $a_{i j}$ is the relative importance of fault $F_{i}$ in regard to $F_{j}$. The value $A_{i j}$ defines the importance (respective values) $F_{i}$ of faults in comparison with $F_{j}$.

Elements $a_{i j}$ are defined by the following rules:

1. If $a_{i j}=\alpha, a_{j i}=1 / \alpha, \alpha \neq 0$.

2. If fault $F_{i}$ has identical relative importance with $F_{j}$, then $a_{i j}=1, a_{j i}=1$, in particular $a_{i i}=1$ for all $i$.

Thus, a back-symmetric matrix A is obtained:

$$
A=\left[\begin{array}{cccc}
1 & a_{12} & \cdots & a_{1 n} \\
1 / a_{12} & 1 & \cdots & a_{2 n} \\
\cdots & \cdots & \cdots & \cdots \\
1 / a_{1 n} & 1 / a_{2 n} & \cdots & 1
\end{array}\right] .
$$

After the representation of quantitative judgements about the fault pairs $\left(F_{i}, F_{j}\right)$ in a numerical expression with the numbers $a_{i j}$, the problem is reduced to that $n$ possible faults $F_{1}, F_{2}, \ldots$, $F_{n}$ will receive a corresponding set of numerical weights $\omega_{1}, \omega_{2}, \ldots, \omega_{n}$, which would reflect the fixed judgements about the condition of the gas turbine subsystem. 


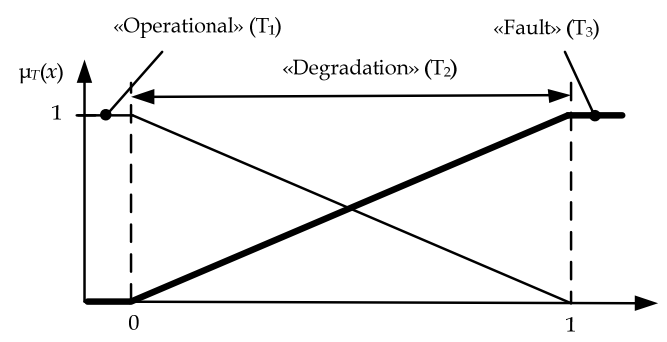

a)

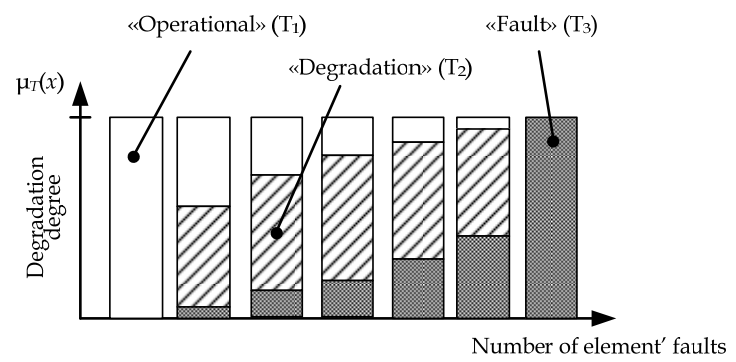

b)

Figure 7. Continuous (a) and discrete (b) scale of degradation

If the expert judgement is absolute at all comparisons for all $i, j, k$, then matrix $A$ is called consistent.

If the diagonal of matrix A consists of units $\left(a_{i j}=1\right)$ and $A$ is the consistent matrix, then at small changes in $a_{i j}$ the greatest eigenvalue $\lambda_{\max }$ is close to $n$, and the other eigenvalue are close to zero.

Based on the matrix of pair comparison values of faults $A$, the vector of priorities for fault classification is obtained, along with vector $\omega$ satisfying the criterion:

$$
A \omega=\lambda_{\max } \omega
$$

where $\omega$ is the eigenvector of matrix $A$ and $\lambda_{\max }$ is the maximum eigenvalue, which is close to the matrix order $n$.

As it is desirable to have the normalized solution, let's slightly change $\omega$, considering $\alpha=\sum_{i=1}^{n} \omega_{i}$ and replacing $\omega$ with $(1 / \alpha) \omega$. This provides uniqueness, and also that $\sum_{i=1}^{n} \omega_{i}=1$. 


$$
A=\underset{F_{2}}{F_{1}}\left[\begin{array}{cccc}
F_{1} & F_{2} & \cdots & F_{n} \\
F_{n} \\
F_{1} / \omega_{1} & \omega_{1} / \omega_{2} & \cdots & \omega_{1} / \omega_{n} \\
\omega_{2} / \omega_{1} & \omega_{2} / \omega_{2} & \cdots & \omega_{2} / \omega_{n} \\
\vdots & \vdots & \vdots & \vdots \\
\omega_{n} / \omega_{1} & \omega_{n} / \omega_{2} & \cdots & \omega_{n} / \omega_{n}
\end{array}\right]\left[\begin{array}{c}
\omega_{1} \\
\omega_{2} \\
\vdots \\
\omega_{n}
\end{array}\right]=\lambda_{\max }\left[\begin{array}{c}
\omega_{1} \\
\omega_{2} \\
\vdots \\
\omega_{n}
\end{array}\right] .
$$

Note that small changes in $a_{i j}$ cause small change in $\lambda_{\max }$ then the deviation of the latter from $n$ is a coordination measure. It allows estimating proximity of the obtained scale to the basic scale of relations. Hence, the coordination index

$$
\left(\lambda_{\max }-n\right) /(n-1)
$$

is considered to be an indicator of "proximity to coordination". Generally, if this number is not greater than 0.1 then it is possible to be satisfied with the judgements about the faults importance.

At each level $h_{i}$ of the hierarchy for $n$ elements of the gas turbine and its subsystems, the state vector \{operational, degradation, fault\} is determined, taking into account the influence coefficients of failures:

$$
S_{h_{i}}\left(x_{n}\right)=\mu_{h_{i}}\left(x_{n}\right) \cdot \omega_{i}
$$

where $\mu_{h_{i}}\left(x_{n}\right)$ is the membership function value of the element $x_{n}$ (degradation degree). To determine the element/unit state of the hierarchy at a higher level $S_{h_{i}}\left(x_{n}\right)$ for the input states of low-level $S_{h_{i-1}}\left(x_{n}\right)$ one stage of defuzzification is performed.

The output value $S_{h_{i}}\left(x_{n}\right)$ is presented in the form of the determined vector of state with parameters $\{$ operational, degradation, fault \}.

The state estimation begins with the bottom level of hierarchy. The description of a state set obtained by means of fuzzification and deffuzification with the use of the logic operations of disjunction $\vee$ (summing), and conjunction $\wedge$ (multiplication), which are designated as follows:

logic operation "AND"

logic operation "OR"

In performing operation "AND" in the inference system of fuzzy logic, the terminal tops are summed in order to determine the general state at one level of hierarchy that is presented as follows:

$$
X_{\Sigma}=x_{1} \vee x_{2} \vee \ldots \vee x_{n}
$$

In performing operation "OR", the "worst" state vector is chosen, with the maximum parameters of degradation $\mu_{\text {degradation }}(x)$ or faults $\mu_{\text {fault }}(x)$. The selector of maximum chooses from the fault influence indexes the one that has the maximum value. 
The use of the hierarchical representation allows a small amount of "short" fuzzy rules to adequately describe multidimensional dependencies between inputs and outputs.

\section{Fuzzy hierarchical Markov state models}

A promising approach to constructing intelligent systems of control, diagnosis and monitoring could be the stochastic modelling on the basis of Markov chains combined with the formalized hierarchy theory.

Within a fuzzy hierarchical model, consider fault development processes with the use of Markov chains. Such dynamic models allow to investigate the change of elements' states in time. Fault development can include not only single faults and their combinations, but also sequences (chains) of so-called "consecutive" faults [10, 11].

During FADEC analysis, classification, formalization and representation of processes of condition monitoring and fault diagnosis for the main subsystems of gas turbines (control, monitoring, fuel supply etc.) is carried out. These processes are represented in the form of Markov chains which allow to analyze the state dynamics of the power-plant.

The transition probability matrix of a Markov chain for modeling faults and their consequences, has a universal structure for all levels of system decomposition (Figure 8):

- system as a whole (power plant);

- constructon units;

- elements.

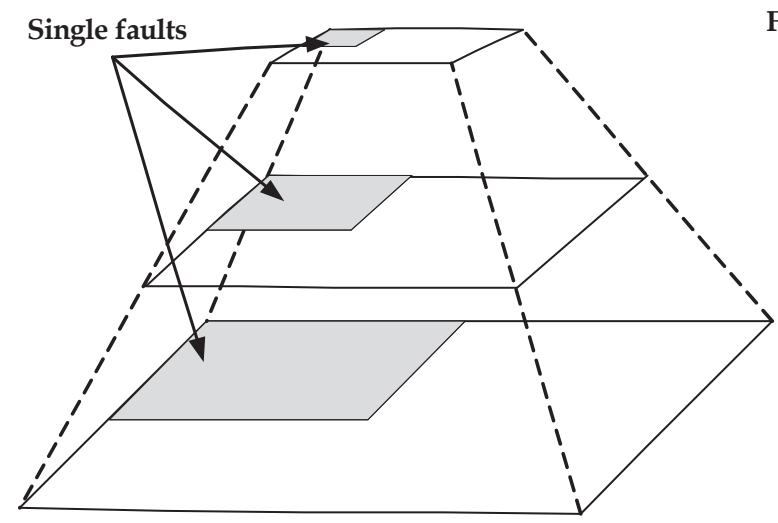

\section{Faults hierarchy}

Power-plant
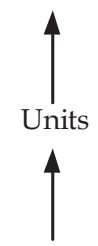

Elements

Figure 8. Hierarchical structure of Markov model of fault

The hierarchical Markov model is built in the generalized state space where physical parameters and binary fault flags are used for the estimation of a state vector of the element, unit 
and power-plant. The state vector includes three parameters \{ operational, degradation, fault \} which allow to track the fault development and degradation process of the system. During FADEC diagnosis, the area of single faults is mostly considered. The proposed Markov model enables to present the system with multiple faults and their sequences. The top state level of a system reflects in the aggregated form the information on faults at the lower state levels.

The elements' state at the levels of the hierarchy depends on the previous values of state parameters of the elements, values of membership functions and fault influence indexes.

For the estimation of transition probabilities between the states of a Markov chain, it is required to calculate relative frequencies of events such as $S_{i} \rightarrow S_{j}$ for a given interval of time. In particular, at the top level, the number of events during one flight (Figure 9) can be of interest.

\begin{tabular}{|l|c|c|c|}
\cline { 2 - 4 } \multicolumn{1}{l|}{$P_{i j}=\operatorname{Prob}\left\{S_{i} \rightarrow S_{j}\right\}$} & 1 & 2 & 3 \\
\hline $\begin{array}{l}\text { 1. Operational } \\
\text { state }\end{array}$ & $P_{11}$ & $P_{12}$ & $P_{13}$ \\
\hline $\begin{array}{l}\text { 2. Degradation } \\
\text { 3. Fault leading to } \\
\text { engine stop }\end{array}$ & $P_{21}$ & $P_{22}$ & $P_{23}$ \\
\hline & & $P_{32}$ & $P_{33}$ \\
\hline
\end{tabular}

Figure 9. Transition probability matrix of power-plant during one flight

The most important events during flight are the emergency turning off of the engine stop (shutdown) and the possibility of its restart. For the probability estimation of such events, it is required to use statistics on all park of the same type engines. For realization of such estimation methods, it is required that flights information was available on each plane and power-plant. Such information should be gathered and stored in a uniform format and should be available for processing. Modern information technologies open possibilities for such research. To analyze fault development processes of one FADEC, it is possible to use results of the automated tests at the hardware-in-the-loop test bed with modeling of various faults and their combinations. In any case, to receive reliable statistical estimates one needs a representative sample of rather large amount of data.

In the analysis of the Markov model, the relation of the transition probability matrix with state of elements and subsystems at each level of hierarchy is considered. Therefore it is nec- 
essary to have the model of the system behavior in various states with various flight condition to guarantee system safety, reliable localization and accommodation of faults.

As the basic mathematical model of the controlled plant, the description in the state space is considered in the form of stochastic difference equations:

$$
X(t+1)=\mathbf{A} X(t)+\mathbf{B} U(t)+\mathbf{F} \xi(t)
$$

where $X \in R^{s}$ is the $s$-dimensioned state vector; $U \in R^{s}$ is the $s$-dimensioned control vector; $\mathrm{A}, \mathrm{B}$ and $\mathrm{F}$ are $(n \times n),(n \times s)$ and $(n \times r)$ matrices accordingly; $\xi \in R^{s}$ is the vector of independent random variables. Thus, the dynamic object described by this finite-difference equation, with input coordinate (control variable) $U$ and output coordinate (state variable) $X$, in the closed scheme of the automatic control system is the controlled Markov process [12,13].

The level of quantisation allows the Markov process to be converted into the Markov chain. Provided $\xi(t)$ is a stationary process, the Markov chain will be homogeneous. Such chain is described by the means of the stochastic transition probability matrix $P$ with the dimensions $(m \times m)$, where $m$ is the number of the chain states. Each element of the matrix $P_{i j}$ represents the probability of the system transition from the condition $X_{i}$ into the condition $X_{j}$ during the time interval $\Delta T$ :

$$
\begin{gathered}
P_{i j}=\operatorname{Prob}\left\{X(t)=X_{i}, X(t+1)=X_{j}\right\}, \quad \forall n \in N, \\
X_{i} \in\left[x_{i}-\frac{\Delta x}{2} ; x_{i}+\frac{\Delta x}{2}\right] ; \quad \sum_{j=1}^{m} P_{i j}=1, i=\overline{1, m} .
\end{gathered}
$$

Condition (2) means that the matrix $P$ should be stochastic and define the full system of events. The sum of elements in each row of the stochastic matrix should equal 1.

The size of the matrix $P$ is defined by the prior information on the order of the object model (1) and the number of the sampling intervals $\Delta x$ and $\Delta u$. The transition probabilities are then estimated as relative frequencies of the corresponding discrete events.

The statistical estimation of the transition probabilities for the controlled Markov chain is performed as the calculation of the frequencies for the corresponding events during observation and the subsequent calculation of the elements of matrix $P$ using the formula:

$$
P_{i j k}=\frac{N_{i j k}}{\sum_{j=1}^{m} N_{i j k}}
$$

where the numerator $N_{i j k}$ is the number of the following events: $\left\{X\left(t_{n}\right)=X_{i}, X\left(t_{n+1}\right)=X_{j}, U\left(t_{n}\right)=U_{k}\right\}$, and the denominator corresponds to the number of 
events such as $\left\{X\left(t_{n}\right)=X_{i}, U\left(t_{n}\right)=U_{k}\right\}$. Thus, for any combination of state $X_{i}$ and control $U_{k}$ a full system of events will consist of the set of the state transitions $X_{j}$.

The normalisation of Equation (3) makes matrix $P$ stochastic. As a result, the set of probabilities in each row $P_{i j}$ describes the full system of events for which the sum of probabilities is equal to unit:

$$
\sum_{j=1}^{m} P_{i j k}=1
$$

The estimation of a transition probability matrix of the Markov model consists of creation of multidimensional histograms which represent an estimate of joint distribution [14, 15].

The use of the hierarchical Markov model allows to "compress" information which has been recorded during one flight, and to present it in a more compact form. In this case, the possibility of analysis and forecast of dynamics of degradation degree (Figure 10) opens. It is possible to analyze the state dynamics of elements and functions at each level of hierarchy in time for decision-making support.

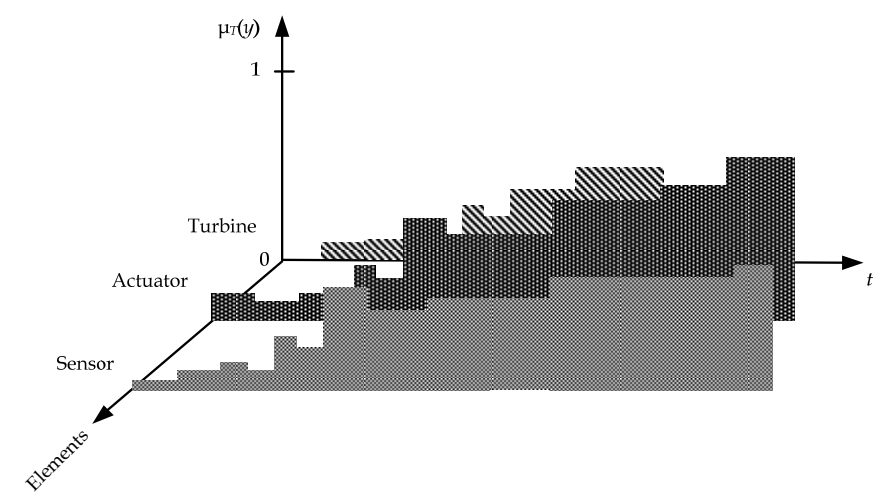

Figure 10. Dynamics of state parameters during flight

The analysis of fault information and state change can be carried out over flight data for the whole duration of maintenance and the whole "fleet" of engines and their systems (Figure 11). Such analysis will assist to increase efficiency for processes of experimental maintenance development and monitoring system support.

Given statistics on all park of engines within several years, it is possible to build empirical estimates of probabilities of the first and second type errors.

Thus, possibilities of application of hierarchical Markov models for the gas turbine and its FADEC for compact representation of information on flight and for the assessment of "sensitivity" of the monitoring system according to actual data are considered. The levels of hierarchy differ with the ways of introducing redundancy and realization of system safety with use of intellectual algorithms of control and diagnosis. Each higher level of hierarchy has 
greater "intelligence" and is designed independently in the assumption of ideal system stability of the lower level.

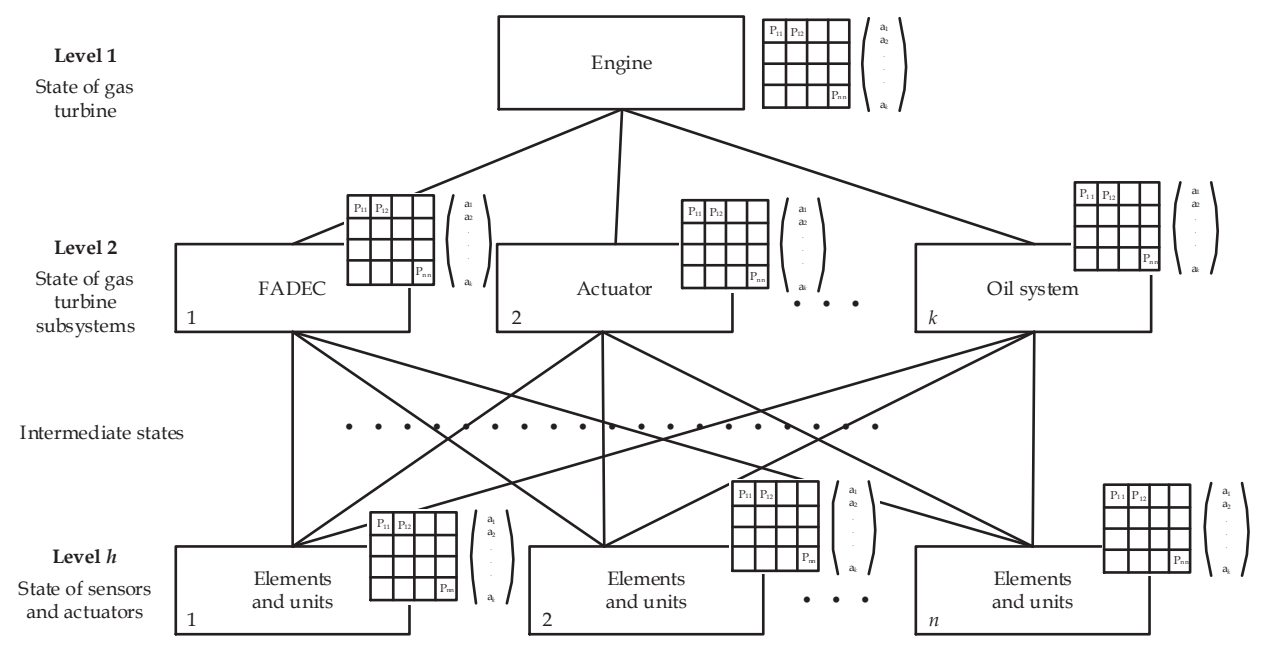

Figure 11. Hierarchical fuzzy Markov model of gas turbine states 
Consider an example. In Figure 12, the FADEC state estimation with faults is presented on the basis of the degradation degree of the elements.

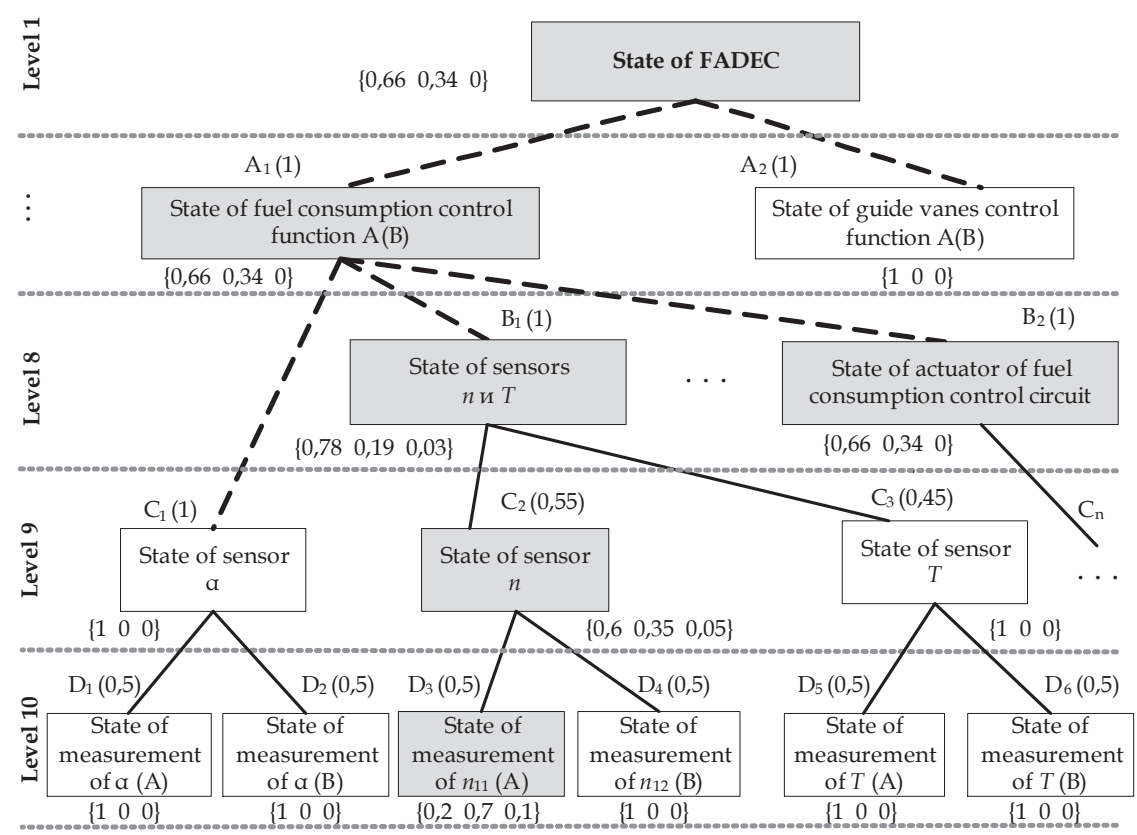

Figure 12. Example of hierarchical estimation of state parameters

At the 10th level the BMS detected a fault of measurement in the form of break of the first coil of parameter $n_{11}$ (shaft speed sensor). On the basis of the fuzzy rule $R^{(2)}$, the parameters of measurement state of $n_{1}$ in the channel $\mathrm{A}$ are characterized by the following three values $\left[\begin{array}{l}\mu_{T_{1}}\left(n_{11}\right)=0,2 \\ \mu_{T_{2}}\left(n_{11}\right)=0,7 \\ \mu_{T_{3}}\left(n_{11}\right)=0,1\end{array}\right]$

$$
\begin{aligned}
& R^{(2)}: \operatorname{IF}\left(n_{11}=A_{2} \text { AND } n_{12}=A_{1}\right) \text { THEN } y=T_{2} \Rightarrow \\
& {\left[\mu_{T_{1}}\left(n_{1}\right)=0,2 ; \quad \mu_{T_{2}}\left(n_{1}\right)=0,7 ; \quad \mu_{T_{3}}\left(n_{1}\right)=0,1\right] .}
\end{aligned}
$$

The measurement state in the channel B is defined as $\left[\begin{array}{l}\mu_{T_{1}}\left(n_{12}\right)=1 \\ \mu_{T_{2}}\left(n_{12}\right)=0 \\ \mu_{T_{3}}\left(n_{12}\right)=0\end{array}\right]$, because no faults were detected. At the 9th level, the sensor state of the $n$ is obtained using the multiplication of the vector of state parameters and faults influence indexes: 


$$
\begin{aligned}
& {\left[\begin{array}{l}
S\left(n_{11}, n_{12}\right)_{\mathrm{o}} \\
S\left(n_{11}, n_{12}\right)_{\mathrm{d}} \\
S\left(n_{11}, n_{12}\right)_{\mathrm{f}}
\end{array}\right]=\left[\begin{array}{l}
\mu_{T_{1}}\left(n_{1}\right)=0,2 \\
\mu_{T_{2}}\left(n_{1}\right)=0,7 \\
\mu_{T_{3}}\left(n_{1}\right)=0,1
\end{array}\right]\left[\begin{array}{l}
\mu_{T_{1}}\left(T_{4}\right)=1 \\
\mu_{T_{2}}\left(T_{4}\right)=0 \\
\mu_{T_{3}}\left(T_{4}\right)=0
\end{array}\right] \times[0,5 ; 0,5]=} \\
& =\left[\begin{array}{l}
(0,2 \times 0,5)+(1 \times 0,5) \\
(0,7 \times 0,5)+(0 \times 0,5) \\
(0,1 \times 0,5)+(0 \times 0,5)
\end{array}\right]=\left[\begin{array}{l}
0,60 \\
0,35 \\
0,05
\end{array}\right] .
\end{aligned}
$$

The state of an element of a higher level is calculated by multiplication of the current state to fault influence indexes of the fault elements. The state of both measurement channels of temperature $T$ is "operational", therefore, the sensor $T$ state equals $\left[\begin{array}{l}S(T)_{\mathrm{o}}=1 \\ S(T)_{\mathrm{d}}=0 \\ S(T)_{\mathrm{f}}=0\end{array}\right]$ The sensor of fuel feed $\alpha$ is also good working.

At the 8th level the state of two sensors $n$ and $T$ after similar calculations becomes equal $\{0,78 ; 0,19 ; 0,03\}$ that indicated the system degradation in the part of control of fuel consumption.

For the estimation of a state of the fuel consumption control function, the "OR" operation is also used. The state of the actuator of fuel consumption control circuit is characterized by the parameters $\{0,66 ; 0,34 ; 0\}$. The state of FADEC is characterized by the fault of fuel consumption control function or the state of the guide vanes control function. Using the operation "OR", the state of FADEC is detected as $\{0,66 ; 0,34 ; 0\}$. In this example, the whole system is considered to be operational, whereas partial degradation is observed, which is not influencing the system operability.

Thus, the technique of state parameters determination for FADEC and its systems on the basis of fuzzy logic and Markov chains is proposed. This technique can be used during flight or in maintenance on the ground.

At the present time a necessary condition for realization of intellectual algorithms is the complete development of the distributed intellectual control models focused on control optimization, forecasting and system safety [16, 17]. In Figure 13, the scheme of the distributed FADEC is shown.

Thus, in each sensor or actuator, it is necessary to have physically built-in control system (or function) to form and monitor the fault signals in the unit, communication lines, cooperating sensors, indication devices and systems [18]. The use of the built-in monitoring control systems working in real time allows to obtain a number of additional possibilities for improving control quality and system operational characteristics as followings:

- emergency states detection of the control object and system;

- fault detection of elements of the control object; 
- state diagnosis and parametrical degradation of the object.

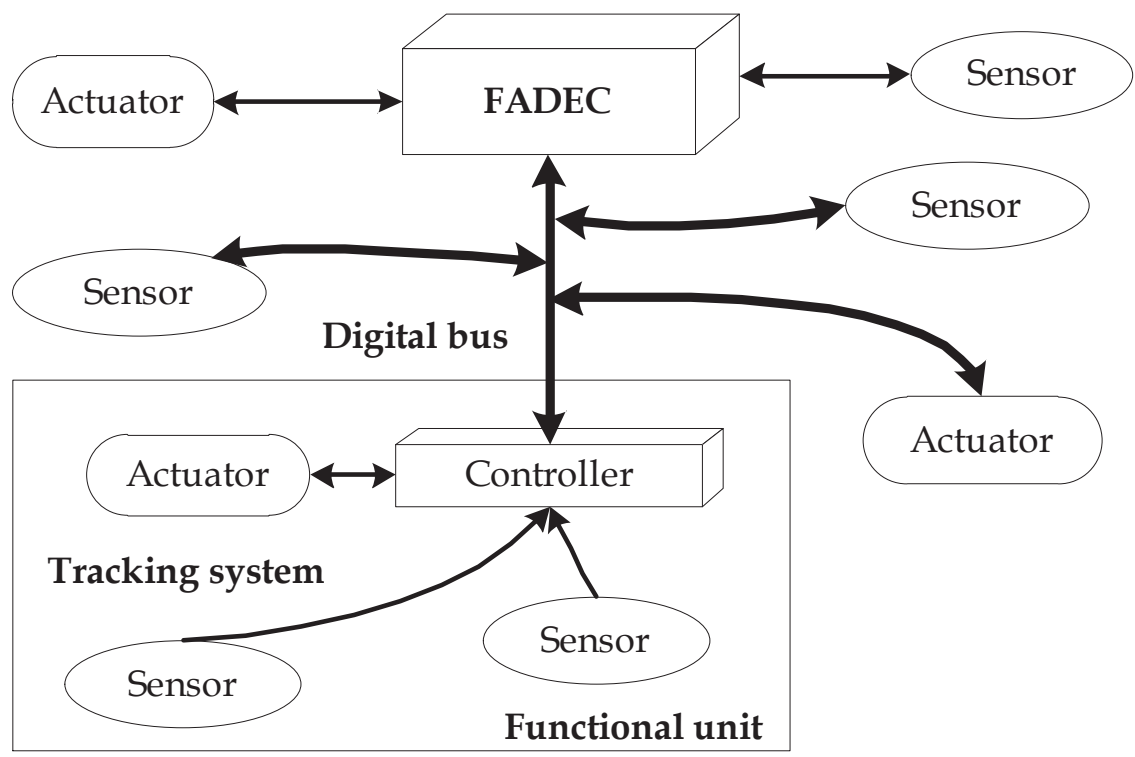

Figure 13. Distributed architecture of FADEC

\section{Conclusion}

In this chapter, the hierarchical fuzzy Markov modeling of fault developments processes has been proposed for the analysis of an airplane system safety. The hierarchical model integrates functional, physical structure of gas turbine and its FADEC elements and units, the tree of states, a tree of fault influence indexes. This model allows to decompose the power-plant for a quantitative estimates of degradation state and gradual faults. The analysis of hierarchies allows to utilize the state model on the basis of fault development processes which estimates the power-plant state at each level of hierarchy. Furthermore, the technique of determination of state parameters of the gas turbine and its systems on the basis of fuzzy logic is presented. The state of each element, unit and system is represented in the form of a vector with parameters \{ operational, degradation, faults \}. The use of the proposed indicator "degradation degree" allows to obtain an objective quantitative estimate of the current state which can be used as, the "distance" to a critical situation and the reserve of time for decision-making in-flight. This indicator is defined on the basis of the discrete-ordered scale and fault influence indexes that allows to determine about $30 \%$ of gradual faults in gas turbine and its systems at the stage of fault development. The examples of fuzzy rules on the basis of expert knowledge are given, whereas fuzzy logic is used for interpolation. 
The application of hierarchical Markov models for the analysis of experimental data is also considered for control system development: as the compact representation of information of a system state change during flight, the estimation of transition probabilities.

\section{Nomenclature}

FADEC - Full Authority Digital Engine Control.

BMS - built-in monitoring system.

$\omega$ - weighting coefficient of fault.

$S$ - state (condition) of gas turbines.

$\mu$ - membership function.

$P$ - probability.

$X$ - state variable.

$U$ - control variable.

\section{Acknowledgements}

The work was supported by the grants from the Russian Foundation for Basic Research (RFFI) №12-08-31279, №12-08-97027 and the Ministry of Education and Science of the Russian Federation.

\section{Author details}

G. G. Kulikov, V. Yu. Arkov and A.I. Abdulnagimov*

*Address all correspondence to: gennadyg_98@yahoo.com; abdulnagimov@gmail.com

Automated Control and Management Systems Department, Ufa State Aviation Technical University, Ufa, Russia

\section{References}

[1] Kulikov GG. Principles of design of digital control systems for aero engines. In Cherkasov BA. (ed.), Control and automatics of jet engines. Mashinostroyeniye, Moscow; 1988. p253-274. 
[2] Arkov VY, Kulikov GG, Breikin TV. Life cycle support for dynamic modelling of gas turbines. Prepr. 15th Triennial IFAC World Congress, Barcelona, Spain; 2002. p2135-2140.

[3] Kuo BC. Automatic control systems. Prentice-Hall: Englewood Cliffs; 1995.

[4] Saaty TL. Analytic Hierarchy Process: Planning, Priority Setting, Resource Allocation. McGraw-Hill, New York, London; 1980.

[5] Saaty TL, Vargas LG. Models, Methods, Concepts \& Applications of the Analytic Hierarchy Process. Kluwer Academic Publisher; 2001.

[6] SAE ARP 4761. Guidelines and Methods for Conducting the Safety Assessment Process on Civil Airborne Systems And Equipment. Aerospace recommended practice; 1996.

[7] Arkov V, Evans DC, Fleming PJ, et al. System identification strategies applied to aircraft gas turbine engines. Proc. 14th Triennal IFAC World Congress; 1999. p145-152.

[8] Kulikov G, Breikin T, Arkov V and Fleming P. Real-time simulation of aviation engines for FADEC test-beds. Proc. Int. Gas Turbine Congress, Kobe, Japan; 1999. p949-952.

[9] Zadeh LA. "Fuzzy algorithms," Information and Control 1968;12(2): 94-102.

[10] Kulikov GG, Fleming PJ, Breikin TV, Arkov VY. Markov modelling of complex dynamic systems: identification, simulation, and condition monitoring with example of digital automatic control system of gas turbine engine. USATU, Ufa; 1998.

[11] Breikin TV, Arkov VY, Kulikov GG. On stochastic system identification: Markov models approach. Proc 2nd Asian Control Conf ASCC'97; 1997. p775-778.

[12] Breikin TV, Arkov VY, Kulikov GG. Application of Markov chains to identification of gas turbine engine dynamic models. International Journal of Systems Science 2006; 37(3) 197-205.

[13] Kulikov G, Arkov V, Lyantsev O, et al. Dynamic modelling of gas turbines: identification, simulation, condition monitoring, and optimal control. Springer, London, New York; 2004.

[14] Kulikov G, Arkov V, Abdulnagimov A. Markov modelling for energy efficient control of gas turbine power plant. Proc. IFAC Conf. on Control Methodologies and Technology for Energy Efficiency, CMTEE-2010, Faro, Portugal; 2010. http:// www.ifac-papersonline.net/Detailed/42981.html

[15] Arkov V, Kulikov G, Fatikov V, et al. Intelligent control and monitoring unit and its investigation using Markov modelling. Proc. IFAC Int. Conf. on Intelligent Control Systems and Signal Processing ICONS-2003, Faro, Portugal; 2003. p489-493 
[16] Vasilyev VI, Ilyasov BG, Valeyev SS. Intelligent Control Systems for gas Turbine engines. Proc. of the Second Scientific Technical Seminar on GT Engines, Turkey, Istanbul; 1996. p71-78.

[17] Culley D, Thomas R and Saus J. Concepts for Distributed Engine Control, AIAA-2007-5709, 43rd AIAA/ASME/SAE/ASEE Joint Propulsion Conference and Exhibit, Cincinnati, Ohio, July 8-11, 2007.

[18] Kulikov GG., Arkov VYu., Abdulnagimov AI. Hierarhical Fuzzy Markov Modelling for System Safety of Power-plant : Proc. of 4th International Symposium on Jet Propulsion and Power Engineering, September 10-12, 2012, Xi' an, China: Northwestern Polytechnical University Press; 2012. p. 589-594. 
